observational studies revealed the benefit of wearing masks (odds ratio $[\mathrm{OR}]=0.13 ; 95 \% \mathrm{CI}: 0.03-0.62)$ and respirators $(\mathrm{OR}=0.12$; 95\% CI: 0.06-0.26) against SARS.

Although Some studies have been against the use of face masks during the COVID-19 pandemic $^{9}$ and one meta-analysis reported that the use of face masks to prevent transmission of influenza virus is still controversial ${ }^{10}$, the mask-wearing policy accompanied with hand hygiene and social distancing appear to have prevented both severe complicated influenza and COVID-19 in Taiwan. In April 2020, the Taiwan Government donated approximately 10 million face masks to countries hit hardest by SARS-COV-2, including the United States and the European Union. We hope that our experience can help other regions to overcome the COVID-19 pandemic as quickly as possible.

Acknowledgement. We thank Professor Ho Pei-Shan, Division of Medical Statistics and Bioinformatics, Department of Medical Research, Kaohsiung Medical University, Kaohsiung, Taiwan.

Contribution. CJ Yang and YH Chen, study design and draft the manuscript. TC Chen, SH Kuo, MH Hsieh, data collection, analysis and discussion.

All authors approved the final manuscript.

Funding. No funding.

Financial support. None.

Conflict of interests. No conflict of interest to be declared.

\section{REFERENCES}

1. Soto DM, Cardona Maya WD, Londono E, Bueno Sanchez JC. The feasibility of generalized face mask usage during COVID-19 pandemic: a perspective from Latin America. Infect Control Hosp Epidemiol 2020:1-4. doi: 10.1017/ ice.2020.227.

2. Yang W, Cao Q, Qin L, Wang X, Cheng Z, Pan A, Dai J, Sun Q, Zhao F, $\mathrm{Qu}$ J, Yan F. Clinical characteristics and imaging manifestations of the 2019 novel coronavirus disease (COVID-19): A multi-center study in Wenzhou city, Zhejiang, China. J Infect 2020;80:388-393. doi: 10.1016/ j.jinf.2020.02.016.

3. Qualls N, Levitt A, Kanade N, Wright-Jegede N, Dopson S, Biggerstaff M, Reed C, Uzicanin A, Group CDCCMGW. Community Mitigation Guidelines to Prevent Pandemic Influenza - United States, 2017. MMWR Recomm Rep 2017;66:1-34. doi: 10.15585/mmwr.rr6601a1.

4. Wong VW, Cowling BJ, Aiello AE. Hand hygiene and risk of influenza virus infections in the community: a systematic review and meta-analysis. Epidemiol Infect 2014;142:922-932. doi: 10.1017/S095026881400003X.

5. Sim SW, Moey KS, Tan NC. The use of facemasks to prevent respiratory infection: a literature review in the context of the Health Belief Model. Singapore Med J 2014;55:160-167. doi: 10.11622/smedj.2014037.

6. Liu X, Zhang S. COVID-19 : Face Masks and Human-to-human Transmission. Influenza Other Respir Viruses 2020 doi: 10.1111/irv.12740.

7. Zhou SS, Lukula S, Chiossone C, Nims RW, Suchmann DB, Ijaz MK. Assessment of a respiratory face mask for capturing air pollutants and pathogens including human influenza and rhinoviruses. J Thorac Dis 2018;10:2059-2069. doi: 10.21037/jtd.2018.03.103.

8. Zhang CQ, Chung PK, Liu JD, Chan DKC, Hagger MS, Hamilton K. Health Beliefs of Wearing Facemasks for Influenza A/H1N1 Prevention: A Qualitative Investigation of Hong Kong Older Adults. Asia Pac J Public Health 2019;31: 246-256. doi: 10.1177/1010539519844082.

9. Bae S, Kim MC, Kim JY, Cha HH, Lim JS, Jung J, Kim MJ, Oh DK, Lee MK, Choi SH, Sung M, Hong SB, Chung JW, Kim SH. Effectiveness of Surgical and Cotton Masks in Blocking SARS-CoV-2: A Controlled Comparison in 4 Patients. Ann Intern Med 2020 doi: 10.7326/M20-1342.

10. Cowling BJ, Zhou Y, Ip DK, Leung GM, Aiello AE. Face masks to prevent transmission of influenza virus: a systematic review. Epidemiol Infect 2010;138:449-456. doi: 10.1017/S0950268809991658.

\title{
Influence of different definitions of central venous catheter-related bloodstream infections on epidemiological parameters in cancer patients
}

\author{
Enrico Schalk $M D^{1}$ (1), Maria J.G.T. Vehreschild $M^{2,3,4}$ and Lena M. Biehl MD ${ }^{2,3}$ \\ ${ }^{1}$ Department of Hematology and Oncology, Otto-von-Guericke University Magdeburg, Medical Center, Magdeburg, Germany, ${ }^{2}$ Department I of Internal Medicine, \\ Faculty of Medicine and University Hospital of Cologne, University of Cologne, Cologne, Germany, ${ }^{3}$ German Center for Infection Research (DZIF), Site Bonn/ \\ Cologne, Cologne, Germany and ${ }^{4}$ Department of Internal Medicine, Infectious Diseases, Goethe University Frankfurt, Frankfurt am Main, Germany
}

To the Editor-Especially for cancer patients, there are multiple coexisting definitions for central venous catheter (CVC)-related bloodstream infections (CRBSIs). Therefore, it is difficult to make comparisons across studies. Furthermore, a considerable number of publications (39 of 190, 21\%) did not report the CRBSI definition or cite a reference for the used definition. ${ }^{1}$ To complicate matters further, guidelines on diagnosis of CRBSIs are subject to change over time. ${ }^{2,3}$ For example, in 2003, the Infectious Diseases Working Party of the German Society of Hematology

\footnotetext{
Author for correspondence: Enrico Schalk, E-mail: enrico.schalk@med.ovgu.de
}

Cite this article: Schalk E, Vehreschild MJGT, and Biehl LM. (2021). Influence of different definitions of central venous catheter-related bloodstream infections on epidemiological parameters in cancer patients. Infection Control \& Hospital Epidemiology, 42: 501-503, https://doi.org/10.1017/ice.2020.274 and Medical Oncology (AGIHO) proposed to distinguish definite (dCRBSIs), probable (pCRBSIs), and possible (possCRBSIs) CRBSIs. ${ }^{4}$ Although these terms are still part of their current guideline, the exact definitions have been adjusted slightly over the years. ${ }^{5,6}$ In addition, dCRBSIs and pCRBSIs are often combined (dpCRBSIs) for reporting purposes. ${ }^{7,8}$

Recently, we provided comparative epidemiological data on CRBSIs from a randomized controlled trial (RCT) and a registry study in high-risk patients with hematological malignancies. ${ }^{7}$ In the RCT data set, the 2008 AGIHO definitions for CRBSIs were initially used, ${ }^{5}$ whereas the registry applied the 2012 AGIHO definitions. $^{6}$ The 2 guidelines differ in their definition of pCRBSIs and possCRBSIs. In brief, criteria for pCRBSIs and possCRBSIs are more strict in the newer guideline (see Supplementary Table S1 for a 
Table 1. Comparison of Epidemiological Parameters of CRBSI According to the 2008 and 2012 AGIHO Criteria

\begin{tabular}{|c|c|c|c|}
\hline Parameter & 2008 AGIHO Definition & 2012 AGIHO Definition & $P$ Value $^{\mathrm{a}}$ \\
\hline \multicolumn{4}{|l|}{ CRBSI rate, $n / N(\%)$} \\
\hline Definite CRBSI & $37 / 613(6.0)$ & $37 / 613(6.0)$ & $1.000^{\mathrm{b}}$ \\
\hline Probable CRBSI & $48 / 613(7.8)$ & $42 / 613(6.9)$ & $.584^{b}$ \\
\hline Possible CRBSI & $151 / 613(24.6)$ & $78 / 613(12.7)$ & $<.001^{\mathrm{b}}$ \\
\hline \multicolumn{4}{|c|}{ CRBSI incidence, $x / 1,000$ CVC days } \\
\hline CRBSI, in total & 21.1 & 14.0 & $<.001^{\mathrm{c}}$ \\
\hline Possible CRBSI & 13.5 & 7.0 & $<.001^{\mathrm{c}}$ \\
\hline
\end{tabular}

Note. AGIHO, Infectious Diseases Working Party of the German Society of Hematology and Medical Oncology; CRBSI, central venous catheter-related bloodstream infection; CVC, central venous catheter.

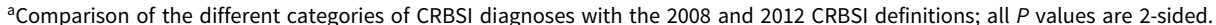

${ }^{b}$ Fisher exact test.

${ }^{c} \chi^{2}$ test.

detailed comparison). Because end points in the $\mathrm{RCT}^{8}$ referred to the 2008 definitions, the underlying data were reassessed according to the 2012 definitions to ensure comparability of the 2 data sets.

The question thus arises: To what extent do different CRBSI definitions influence reported CRBSI epidemiology? Therefore, in this study, we compared the epidemiological data of CRBSIs of the aforementioned RCT once using the 2008 definitions and once the 2012 definitions.

In total, 613 CVCs from cancer patients were analyzed. The patient characteristics were reported elsewhere. ${ }^{8}$ Our results are summarized in Table 1. According to the 2008 definitions, 236 of 613 CVCs (38.5\%) fulfilled criteria for a CRBSI in comparison to 157 of 613 CVCs $(25.6 \%)$ according to the newer definitions $(P<.001)$. This difference was attributable to documentation of significantly more possCRBSIs in the assessment with the older definitions (151 of 613 [24.6\%] vs 78 of 613 [12.7\%]; $P<.001$ ), but we detected no significant differences for dCRBSIs, pCRBSIs, or dpCRBSIs.

Like the CRBSI rate, the total CRBSI incidence and the incidence of possCRBSIs differed significantly between the assessments with the 2008 and the 2012 definitions (see Table 1 for details).

To measure the interrater reliability (interobserver agreement, precision) between the 2 assessments, we calculated Cohen's $\kappa .{ }^{9}$ Taking all 613 CVCs into account, there was a strong agreement $(\kappa=.84)$ between the 2008 and the 2012 definitions. However, this was mostly due to the high proportion of CVCs without any CRBSI diagnosis (311 of 613, 61.5\%), which were consistently classified in both the application of the 2008 and the 2012 definitions. Furthermore, all 37 dCRBSIs classified according to the 2008 definitions also met the 2012 definitions for dCRBSIs $(\kappa=1.00$, perfect agreement). As expected, classifications into the group of pCRBSIs and possCRBSIs were less consistent between the 2 definitions. Only 22 of 48 (45.8\%) of the older definition of pCRBSIs were also classified as pCRBSIs according to the newer definition $(\kappa=.71$, moderate agreement); 26 of $48(54.2 \%)$ were downgraded to possCRBSIs. Analyzing the 85 cases in the combined group of
dpCRBSIs according to the 2008 definitions, 59 (69.4\%) met the same criteria in the 2012 definitions ( $\kappa=.82$, strong agreement). Of 151 possCRBSIs in the assessment with the 2008 definitions, only $52(34.4 \%)$ met the criteria for possCRBSIs according to the newer definitions $(\kappa=.47$, weak agreement); 79 of 151 (52.3\%) were downgraded to cases without a CRBSI diagnosis, whereas 20 of $151(13.2 \%)$ were upgraded to pCRBSIs.

The CRBSI incidence reported in the literature varies strongly, among other factors, depending on the applied CRBSI definition. An accurate CRBSI diagnosis is important for effective and timely treatment and, thus, reduction of further complications. In cancer patients, CRBSI rates are an important end point for supportive care. ${ }^{1}$ Consensus regarding CRBSI definitions is a prerequisite for a meaningful comparison of this important outcome parameter. In a previous study, analyzing the same CVC cohort based on criteria with a microbiological focus yielded a 46\% lower CRBSI incidence compared to the incidence analyzed on the basis of a more clinically oriented definition. ${ }^{10}$ Similarly, in our comparison of CRBSI definitions of the former and the updated AGIHO guideline, the CRBSI incidence was $~ 34 \%$ lower using the stricter microbiological criteria (2012 AGIHO definition) compared to the assessment with the 2008 AGIHO definition. However, this discrepancy was only due to the large proportion of possCRBSIs, that is, the more clinically assessed CRBSIs. The interrater reliability regarding dpCRBSIs showed strong agreement, and no significant differences were detected in the dpCRBSIs rate and incidence between the 2008 and 2012 definitions. Based on these findings, strict microbiological definitions seem to yield more consistent epidemiological parameters; they should be used in future studies.

Supplementary material. To view supplementary material for this article, please visit https://doi.org/10.1017/ice.2020.274

Acknowledgments. The authors would like to thank all COAT investigators involved in the conduct of the randomized controlled trial.

Financial support. No financial support was provided relevant to this article. 
Conflicts of interest. M.J.G.T.V. has served at the speakers' bureau of Akademie für Infektionsmedizin, Ärztekammer Nordrhein, Astellas Pharma, Basilea, Gilead Sciences, Merck/MSD, Organobalance, and Pfizer, received research funding from 3M, Evoinik, Glycom, Astellas Pharma, DaVolterra, Gilead Sciences, MaaT Pharma, Merck/MSD, Morphochem, Organobalance, and Seres Therapeutics and is a consultant to Alb-Fils Kliniken $\mathrm{GmbH}$, Arderypharm, Astellas Pharma, Ferring, DaVolterra, MaaT Pharma, and Merck/MSD. L.M.B. reports lecture honoraria from Astellas, and MSD and travel grants from $3 \mathrm{M}$ and Gilead. E.S. reports no conflicts of interest relevant to this article.

\section{References}

1. Tomlinson D, Mermel LA, Ethier MC, Matlow A, Gillmeister B, Sung L. Defining bloodstream infections related to central venous catheters in patients with cancer: a systematic review. Clin Infect Dis 2011:697-710.

2. Mermel LA, Farr BM, Sherertz RJ, et al. Guidelines for the management of intravascular catheter-related infections. Clin Infect Dis 2001;32: 1249-1272.

3. Mermel LA, Allon M, Bouza E, et al. Clinical practice guidelines for the diagnosis and management of intravascular catheter-related infection: 2009 update by the Infectious Diseases Society of America. Clin Infect Dis 2009;49:1-45.

4. Fätkenheuer G, Buchheidt D, Cornely OA, et al. Central venous catheter (CVC)-related infections in neutropenic patients-guidelines of the Infectious Diseases Working Party (AGIHO) of the German Society of
Hematology and Oncology (DGHO). Ann Hematol 2003;82 suppl 2: S149-S157.

5. Wolf HH, Leithauser M, Maschmeyer G, et al. Central venous catheterrelated infections in hematology and oncology: guidelines of the Infectious Diseases Working Party (AGIHO) of the German Society of Hematology and Oncology (DGHO). Ann Hematol 2008;87:863-876.

6. Hentrich M, Schalk E, Schmidt-Hieber M, et al. Central venous catheterrelated infections in hematology and oncology: 2012 updated guidelines on diagnosis, management and prevention by the Infectious Diseases Working Party of the German Society of Hematology and Medical Oncology. Ann Oncol 2014;25:936-947.

7. Schalk E, Teschner D, Hentrich M, et al. Central venous catheter-related bloodstream infections in patients with hematological malignancies: comparison of data from a clinical registry and a randomized controlled trial. Infect Control Hosp Epidemiol 2020;41:254-256.

8. Biehl LM, Huth A, Panse J, et al. A randomized trial on chlorhexidine dressings for the prevention of catheter-related bloodstream infections in neutropenic patients. Ann Oncol 2016;27:1916-1922.

9. McHugh ML. Interrater reliability: the kappa statistic. Biochem Med (Zagreb) 2012;22:276-282.

10. Tribler S, Brandt CF, Hvistendahl M, et al. Catheter-related bloodstream infections in adults receiving home parenteral nutrition: substantial differences in incidence comparing a strict microbiological to a clinically based diagnosis. J Parenter Enteral Nutr 2018;42:393-402.

\title{
Regional and statewide antibiograms as targeted interventions against antibiotic resistance
}

\author{
John G Plante BS ${ }^{1}$, Hana R Winders PharmD2 (1), P. Brandon Bookstaver PharmD ${ }^{2,3}$ (D), Majdi N Al-Hasan MBBS ${ }^{1,4}$ (D), \\ Julie Ann Justo PharmD, $\mathrm{MS}^{2,3}$ (D), Katie $\mathrm{S}$ Waites $\mathrm{MPH}^{5}$ and Sharon Weissman MD ${ }^{1,4}$ \\ ${ }^{1}$ University of South Carolina School of Medicine, Columbia, South Carolina, ${ }^{2}$ Department of Clinical Pharmacy and Outcomes Sciences, University of South \\ Carolina College of Pharmacy, Columbia, South Carolina, ${ }^{3}$ Department of Pharmacy, Prisma Health Richland, Columbia, South Carolina, ${ }^{4}$ Department of \\ Medicine, Prisma Health University of South Carolina Medical Group, Columbia, South Carolina and ${ }^{5}$ South Carolina Department of Health and Environmental \\ Control, Columbia, South Carolina
}

To the Editor-Antibiotic resistance is becoming an increasingly heavy burden on our nation, leading to significant patient morbidity, mortality, and healthcare expenditures. ${ }^{1}$ Antibiotic misuse may be considered a primary driver of resistance, and recent studies suggest that $\sim 20 \%-30 \%$ of inpatient antibiotics and $30 \%-40 \%$ of ambulatory antibiotics were inappropriately prescribed. ${ }^{2,3}$ According to the Centers for Disease Control and Prevention's (CDC's) 2019 Threats Report, ${ }^{1}>2.8$ million resistant infections and 35,000 associated deaths are reported annually in the United States. Although this has been accompanied by a $27 \%$ reduction in the number of resistant nosocomial infections, the total number of annual resistant infections has increased, highlighting the need for additional community-focused antimicrobial stewardship interventions. ${ }^{1}$

The impact of several statewide and regional antibiograms on clinical management and stewardship efforts has previously been described. ${ }^{4,5}$ Here, we discuss regional and statewide antibiograms in South Carolina. The Antimicrobial Stewardship Collaborative of

Author for correspondence: Hana R. Winders, Email: hwinders@cop.sc.edu

Cite this article: Plante JG, et al. (2021). Regional and statewide antibiograms as targeted interventions against antibiotic resistance. Infection Control \& Hospital Epidemiology, 42: 503-505, https://doi.org/10.1017/ice.2020.273
South Carolina (ASC-SC) was established in 2016 with support from the CDC. This organization coordinates a variety of statewide antimicrobial stewardship initiatives, including the statewide antibiogram project presented in this letter. This endeavor represents the continuation of a smaller-scale pilot project conducted from 2007 to 2011 through a collaboration between the South Carolina Department of Health and Environmental Control and the University of South Carolina College of Pharmacy.

From 2007 to 2011 and from 2015 to 2017, hospitals and nursing homes throughout South Carolina were asked to submit their annual facility-specific and ambulatory antibiograms to ASC-SC. Each antibiogram was deconstructed into individual isolates and combined into one statewide and multiple regional antibiograms annually. Most of the data consisted of isolates from acute-care hospitals. The yearly cumulative antibiograms were redistributed for use by healthcare facilities across the state.

The compiled statewide antibiogram contains 2017 isolate data from 49 institutions (Fig. 1). Statewide susceptibility rates for the 2017 year were compared to the 2015 year. We used $\chi^{2}$ analysis to assess significance at an $\alpha$ level of 0.05 .

Overall, Escherichia coli was the most frequently reported organism (33,848 isolates in 2017). From 2015 to 2017, Acinetobacter baumannii demonstrated increased susceptibility 